\title{
The ethics of personal genetic profiling
}

\section{CHRISTOPHER HOOD ${ }^{1}$}

Direct-to-consumer personal genetic profiling services that claim to predict people's individual disease risks may promise a new era of 'personalised healthcare', but a report from the Nuffield Council on Bioethics has found that the results are often inconclusive and more evidence should be provided by the companies who sell them.

In September 2008, the Council established a Working Party, which I chaired, to consider the ethical issues raised by developments in medical profiling and online medicine. One of our primary tasks was to investigate the burgeoning market for personal genetic profiling services.

Two years on, the Council has published its findings. The resulting report ${ }^{2}$ raises a number of concerns about personal genetic profiling services and puts forward a series of policy recommendations to address these concerns.

\section{Background}

Whilst the NHS provides genetic tests to detect rare monogenic disorders, such tests are strictly regulated and genetic counselling is provided to help people decide whether to take the tests and to help them interpret the results. The conditions that are screened for must fit a number of criteria including that the condition must be serious, and effective treatment or intervention must be available.

In contrast, personal genetic profiling services are marketed by private companies to healthy people as a way of finding out about their genetic risk of developing various common diseases in the future. Clients pay up to US\$2,000 to send a DNA sample for analysis and receive back a list of specific risk predictions for a range of multifactorial conditions such as heart disease, diabetes and several types of cancer.

Currently there is no overarching regulation of commercial DNA profiling services, although some aspects are regulated - for example advertising on websites is regulated by such bodies as the Advertising Standards Authority in the UK.

The Council's first concern with these services is that the reliability of the estimated lifetime risk statistics produced for each disease is questionable. Both academic research and journalistic investigation has shown that when an individual submits a DNA sample to different companies, they can get quite different estimates for the same diseases.

Furthermore, even if they are reliable, in almost all cases the level of increase or decrease in risk that can be predicted is very small. These are highly complex disorders and whether or not a person will develop the disease depends not only on 
the specific interaction of multiple genes but also on other factors including the person's lifestyle and environment. We concluded that that there is currently insufficient evidence to substantiate claims that these tests provide an accurate and reliable indication of a person's risk of developing these types of disease.

In addition, the estimated lifetime risk statistics that people receive from these tests are difficult for non-specialists to interpret, particularly without the advice of a genetic counsellor or doctor.

Another concern is the way that these tests are marketed, which may lead people to believe that they will be able to take control of their future health after receiving their genetic profile by changing their lifestyle or seeking advance treatments. However, the promise of people being able to 'take control' of their health is misleading. Scientists agree that for these kinds of complex disorders we are a long way from being able to adapt medical treatments to individual genotypes. Beyond making obvious health choices such as eating a balanced diet and cutting back on alcohol (which people will be aware of with or without these tests), in the vast majority of cases there is probably little that people can do to change their risks.

Despite these concerns about personal genetic profiling, one of the problems encountered during our investigation is that at this time, there is very little evidence available both about the number of people buying these services, and the harms (or benefits) they experience from taking the tests.

Given this lack of evidence, it would be unjustified to recommend banning these services altogether. Any intervention should be proportionate to the problem, and less coercive interventions should be explored first (unless the degree of harm in a particular case merits a more stringent type of intervention). We also recognise the difficulties involved with regulating international trade via the internet and therefore our recommendations place emphasis on measures that will help individuals assess the reliability of the websites used to advertise and sell these services.

\section{Ethical and social considerations}

Personal genetic profiling is just one of a range of developments that are promised by their providers as leading to a new era of 'personalised healthcare', and we found several meanings for 'personalised'. For example, it can mean healthcare that is tailored to a person's specific characteristics, or healthcare where more responsibility is given to individuals rather than medical professionals.

Some of the questions around personalised healthcare developments concern their interaction with two key social pressures, 'responsibilisation' and 'consumerisation'. These pressures give rise to some of the ethical challenges associated with personal genetic profiling. 
The idea of 'responsibilisation' emerges when we consider that people are now actively encouraged to take more responsibility for their own health, to lead a healthy lifestyle and play an active role in managing their healthcare. Developments in medical profiling and online medicine can provide new tools to enable people to take more responsibility for their health. These are good in principle, but the idea of increasing personal responsibility in the context of personal genetic tests is somewhat double edged, Feeling responsible for a disease that cannot be prevented may induce misplaced feelings of guilt.

When thinking about 'consumerisation', there is nothing new about being able to select and pay for healthcare in the private sector, but today even public healthcare services are aiming to become more user focused. A consumerist approach to healthcare and the new technologies available has been seized upon by providers of personal genetic testing.

The Council identified five ethical values that should be considered when making recommendations about new technologies in medical profiling and online medicine, including personal genetic profiling. These were:

- Private information should be safeguarded.

- Individuals should be able to pursue their own interests in their own way.

- The state should act to reduce harm.

- Public resources should be used fairly and efficiently.

- Social solidarity (sharing risks and working together to protect the vulnerable) should inform public policy.

These ethical values often conflict with one another but all are important and no one value 'trumps' another. When applying these values to the dilemmas raised by personal genetic profiling, we attempt to 'soften' the conflicts by respecting each value as much as possible, and making recommendations that are evidence-based, proportionate and feasible.

\section{Recommendations}

\section{Information provided by commercial providers}

One of our key recommendations is that companies who provide these services should give people clearer and more prominent information before they buy. The information should explain more about the limitations of the results, the fact that they may require interpretation by a doctor or geneticist, and the possible implications of taking one of 
these tests, such as whether to tell family members who may also be affected by the results.

\section{Claims made about genetic profiling tests}

UK regulators should request clear evidence for any claims being made by companies about the clinical value of the genetic tests they provide.

\section{Information available to consumers}

Government-provided health websites should include general information for the public about the risks and benefits associated with personal genetic profiling services. This information should include reference to any difficulties with establishing clinical validity, the possibility of finding out about conditions for which treatment is not available, and whether it could be necessary for consumers to inform life, mortgage or travel insurance companies of the results of any tests, either at the time or in the future.

Given the lack of available evidence at the current time, independent research on the health and psychological impact and effects of multifactorial genetic susceptibility testing on individuals, including children, should be carried out by public healthcare systems.

\section{Impacts for the public healthcare system}

People who take these tests may subsequently wish to discuss their genetic profile doctor and seek advice about how it may affect them in the future. Doctors should receiving training on how to advise people and making referral decisions based on these tests.

\section{Testing children}

In the case of children, given our ethical value of the state striving to reduce harm, we recommend that companies should only analyse the DNA of children if (i) a genetic test meets the criteria of the UK National Screening Committee and (ii) valid parental consent has been given.

\section{What's next?}

During our investigation we found that the scientific community remains divided on the issue of how long it will take for the results derived from individual genetic profiling to prove useful for tailoring lifestyle and medical interventions. However, as the cost of sequencing DNA falls, finding out about personal genetic information is likely to become an affordable possibility for more and more people. That is why we need more research on the impact of such tests, and for better information to be 
GSP Genomics, Society and Policy

online 2010, Vol.6, No.1 pp.61-65

provided for potential users to help them better understand the implications and limitations.

\footnotetext{
${ }^{1}$ Professor of Government at the University of Oxford and Chair of the Nuffield Council on Bioethics Working Party on Medical Profiling and Online Medicine

${ }^{2}$ Nuffield Council on Bioethics 2010 Medical profiling and online medicine: the ethics of personalised healthcare in a consumer age (London: Nuffield Council on Bioethics) available at www.nuffieldbioethics.org/personalised
} 